\title{
Engineering anomalous quantum Hall plateaus and antichiral states with ac fields
}

\author{
Álvaro Gómez-León, ${ }^{1,3,4}$ Pierre Delplace, ${ }^{2}$ and Gloria Platero ${ }^{1}$ \\ ${ }^{1}$ Instituto de Ciencia de Materiales, CSIC, Cantoblanco, Madrid E-28049, Spain \\ ${ }^{2}$ Département de Physique Théorique, Université de Genève, CH-1211 Genève, Switzerland \\ ${ }^{3}$ Department of Physics \& Astronomy, University of British Columbia, Vancouver, British Columbia V6T 1Z1, Canada \\ ${ }^{4}$ Pacific Institute of Theoretical Physics, Vancouver, British Columbia V6T 1Z1, Canada \\ (Received 5 February 2014; revised manuscript received 23 April 2014; published 7 May 2014)
}

\begin{abstract}
We investigate the ac electric field induced quantum anomalous Hall effect in honeycomb lattices and derive the full phase diagram for arbitrary field amplitude and phase polarization. We show how to induce antichiral edge modes as well as topological phases characterized by a Chern number larger than 1 by means of suitable drivings. In particular, we find that the Chern number develops plateaus as a function of the frequency, providing a time-dependent analog to the ones in the quantum Hall effect.
\end{abstract}

DOI: 10.1103/PhysRevB.89.205408

PACS number(s): 73.20.-r, 03.65.Vf, 71.10.Pm, 71.20.-b

Introduction. The realization of different topological states of matter is one of the major challenges for both fundamental reasons and technological perspectives. Several of these states have been originally predicted in the honeycomb lattice, whose Dirac-like band structure brings the system at a critical point of a topological phase transition. There, the development of gaps by different mechanisms results in a variety of topological phases $[1,2]$. In this line, the quantum spin Hall phase was first obtained in HgTe quantum wells [3,4], where the topological phase transition was controlled by the thickness of the quantum well. Likewise, the quantum anomalous Hall effect (QAHE) - for which time-reversal symmetry (TRS) is broken in the absence of a magnetic field-was originally proposed by Haldane in the honeycomb lattice [1]. However, despite the success of the theoretical model, it has been very difficult to achieve experimentally, until very recently in doped topological insulators [5].

Simultaneously, different techniques have been proposed to externally control the topological properties of a system. One of the most promising consists in periodically driving the system in order to achieve a topological phase transition [6-11]. Proposals with a time periodic driving in semiconductors $[6,12]$, optical lattices [13], or graphene [7,14-18] have been suggested to achieve various dynamical generalizations of static topological phases, called Floquet topological phases [12], and have been recently observed in photonic crystals [19]. Importantly for our purposes, the previous studies for the honeycomb lattice were restricted either to numerical calculations in finite-size systems, where the physical mechanism driving the topological phase transitions was not clear, or to very high frequencies and low-energy approximations, where most of the phase diagram remained unknown.

In the present work, we derive the full phase diagram of periodically driven honeycomb lattices in the QAHE regime, by explicitly calculating the Chern number of the Floquet bands. Our model is valid for arbitrary field frequency, amplitude, and polarization and therefore goes beyond the single Dirac cone description and the rotating wave approximation.

Surprisingly, we find that a clockwise driving may also lead to counterclockwise (or antichiral) edge states, and demonstrate that their appearance is linked to two distinct band inversion mechanisms. Moreover, we show how to induce topological phases with Chern numbers larger than 1, and discuss the emergence of the chiral and antichiral edge states for different boundary conditions. Finally, we find that the Chern number, as a function of the frequency, develops a plateau structure which provides a Floquet analog of the quantum Hall effect plateaus.

Model. We consider noninteracting spinless particles in a honeycomb lattice, coupled to an in-plane, time-dependent, spatially homogeneous vector potential $\mathbf{A}(\tau)$ of period $T=$ $\frac{2 \pi}{\omega}$, and formulate the problem within the Floquet formalism. In general, the hopping parameters to the $j$ first neighbors in the presence of an ac field read $t_{j}(\tau)=t e^{i \mathbf{A}(\tau) \cdot \mathbf{d}_{j}}$. Due to the time and spatial periodicities, the system is described by Floquet-Bloch states which fulfill the Floquet eigenvalue equation: $\mathcal{H}(\mathbf{k}, \tau)\left|u_{\alpha, \mathbf{k}}(\tau)\right\rangle=\epsilon_{\alpha, \mathbf{k}}\left|u_{\alpha, \mathbf{k}}(\tau)\right\rangle$, where $\mathcal{H}(\mathbf{k}, \tau) \equiv$ $H(\mathbf{k}, \tau)-i \partial_{\tau}$ is the Floquet operator, $H(\mathbf{k}, \tau)$ the timedependent Bloch Hamiltonian, $\alpha$ the Floquet band index, $\left|u_{\alpha, \mathbf{k}}(\tau)\right\rangle$ the $T$-periodic Floquet-Bloch states, and $\epsilon_{\alpha, \mathbf{k}}$ the quasienergy which is defined modulo $\omega$. In order to calculate the quasienergies it is useful to rewrite $\mathcal{H}(\mathbf{k}, \tau)$ in terms of its Fourier components. In general they are given by (details in Appendix A)

$$
\left\langle\left\langle u_{\alpha, \mathbf{k}, p^{\prime}}|\mathcal{H}(\mathbf{k}, \tau)| u_{\beta, \mathbf{k}, p}\right\rangle\right\rangle=\tilde{t}_{p^{\prime}, p}^{\alpha, \beta}-p \omega \delta_{p^{\prime}, p} \delta_{\alpha, \beta},
$$

where $\langle\langle\ldots\rangle\rangle$ is a composed scalar product which includes a time average, and the hoppings between Fourier components $\tilde{t}_{p^{\prime}, p}^{\alpha, \beta}$ are given in terms of the undriven hoppings:

$$
\tilde{t}_{p^{\prime}, p}^{\alpha, \beta} \equiv \frac{1}{T} \int_{0}^{T} \sum_{j} e^{i \omega \tau\left(p^{\prime}-p\right)} e^{i \mathbf{k} \cdot \mathbf{d}_{j}} t_{j}^{\alpha, \beta}(\tau) d \tau .
$$

For a honeycomb lattice $\mathbf{d}_{1}=a(1,0), \mathbf{d}_{2}=a\left(-\frac{1}{2}, \frac{\sqrt{3}}{2}\right), \mathbf{d}_{3}=$ $a\left(-\frac{1}{2},-\frac{\sqrt{3}}{2}\right)$ with $a$ the intersite spacing. Then the Fourier components of the time-dependent hoppings are $\left(q=p^{\prime}-p\right)$

$$
\tilde{t}_{p^{\prime}, p}=H_{q}(\mathbf{k})=\left(\begin{array}{cc}
0 & \rho_{q}(\mathbf{k}) \\
\rho_{-q}^{*}(\mathbf{k}) & 0
\end{array}\right),
$$

with $\rho_{q}(\mathbf{k})=\sum_{j} t_{j, q}^{F} e^{i \mathbf{k} \cdot \mathbf{a}_{j}}, \quad \mathbf{a}_{1}=0, \quad \mathbf{a}_{2}=a\left(\frac{3}{2}, \frac{\sqrt{3}}{2}\right), \quad \mathbf{a}_{3}=$ $a\left(\frac{3}{2},-\frac{\sqrt{3}}{2}\right)$. Finally, for a vector potential of the form $\mathbf{A}(\tau)=$ $\left(A_{x} \sin (\omega \tau), A_{y} \sin (\omega \tau+\phi), 0\right)$, with $\phi$ the phase difference which takes into account the in-plane rotation of the field, the renormalized dressed hoppings read $t_{j, q}^{F}=t e^{i q \Psi_{j}} J_{q}\left(\mathcal{A}_{j}\right)$ [20], 
where $J_{q}$ denotes the $q$ th Bessel function of the first kind, and the functions $\Psi_{j}$ and $\mathcal{A}_{j}$ encode all the information of the ac field configuration, being $\Psi_{1}=0$ and

$$
\begin{aligned}
\tan \Psi_{2,3} & =\mp \frac{\sqrt{3} A_{y} \sin (\phi)}{A_{x} \mp \sqrt{3} A_{y} \cos (\phi)}, \\
\mathcal{A}_{1} & =A_{x} a, \\
\mathcal{A}_{2,3} & =\frac{a}{2} \sqrt{A_{x}^{2}+3 A_{y}^{2} \mp 2 \sqrt{3} A_{x} A_{y} \cos (\phi)} .
\end{aligned}
$$

We stress that the previous derivation is not restricted to a single Dirac cone and is valid for any field polarization, frequency, and intensity.

In physical realizations of honeycomb systems, additional Bloch bands are present as for instance the sigma bands in graphene, the higher energy modes in the wave guides of photonic crystals, or in the harmonic confinement potential of the trapped cold atoms. These additional bands are specific to each realization of honeycomb lattices and their treatment is out of the scope of the present work.

Phase diagram of the lattice model. In the high-frequency regime $(\omega \gg t)$ the Floquet operator [Eq. (1)] is approximately block diagonal, meaning that the absorption/emission of photons with energy $\omega$ is very unlikely. Then, one can choose a single block as the effective Floquet operator (note that all blocks are equivalent, and related by a shift $p \omega$, with $p$ an integer). In this limit, the system fulfils both time-reversal and particle-hole symmetry, and its Hamiltonian is analogous to the one of an undriven honeycomb lattice with renormalized anisotropic hoppings $t_{j, 0}^{F}=t J_{0}\left(\mathcal{A}_{j}\right)$. The hopping anisotropy breaks $C_{3}$ symmetry and changes the position of the Dirac points. When the renormalized hoppings fulfill $\pm t_{i, 0}^{F} \pm t_{j, 0}^{F} \pm t_{k, 0}^{F}=0 \quad(i \neq j \neq k)$, the Dirac points merge, leading to an insulating state. Such an insulating phase may support nondispersive zero-energy edge modes, and when this happens we name it the Zak insulating phase [20] (a calculation for these states is shown in Appendix C). Figure 1 (top) shows the phase diagram and the variety of Dirac semimetalic (SM) and Zak insulating (ZI) phases that can be obtained as a function of the electric field amplitude $E$ (whose relation with the vector potential is given by $\mathbf{E}=-\partial_{t} \mathbf{A}$ ) and the field polarization $\phi$. The calculation of the spectrum in a finite-size system exhibits nondispersive zero-energy edge modes, as we show in Appendix C.

Importantly, even at high frequency, a small coupling between Floquet sidebands exists, and if this coupling breaks TRS - as for the case of nonlinearly polarized fields-an inverted gap opens [14]. This can be understood in terms of the appearance of nonzero complex phases $\Psi_{i}$ attached to the renormalized hoppings for nonlinearly polarized fields. In order to take into account the effect of this small coupling, we now use first-order perturbation theory in $t / \omega$ and derive an effective time-independent Bloch Hamiltonian for the steady state (details in Appendix B):

$$
H_{\text {eff }}(\mathbf{k})=H_{0}-\frac{1}{\omega}\left(\left[H_{0}, H_{-1}\right]-\left[H_{0}, H_{1}\right]+\left[H_{-1}, H_{1}\right]\right) .
$$

The correction to $H_{0}(\mathbf{k})$ is proportional to $t^{2} \sigma_{z} / \omega$, where $\sigma_{z}$ is the Pauli matrix, and opens a dynamical gap at the Dirac points for $\phi \neq 0, \pi$. The $k$ dependence of this correction yields

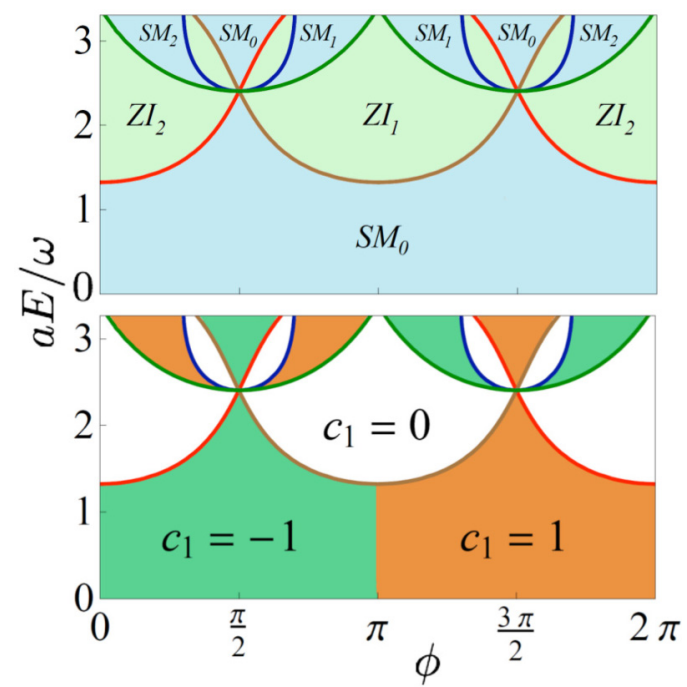

FIG. 1. (Color online) Top: Phase diagram for the unperturbed Hamiltonian $H_{0}$ with $A_{x}=A_{y}$ as a function of the electric field amplitude $E$ and the field polarization $\phi$. It shows semimetallic $\mathrm{SM}_{i}$ (light blue) and insulating phases $\mathrm{ZI}_{i}$ (light green), labeled with a subindex $i$ which accounts for the inequivalent directions along the FBZ where the Wilson loop is nonzero. The different phases are obtained by the merging of the Dirac points at the different time reversal symmetric points of the FBZ. Bottom: First Chern number values at the lower Floquet band for $\omega=10 t$, when first-order coupling among the side bands is considered (green: $c_{1}=-1$, white: $c_{1}=0$; orange: $c_{1}=1$ ).

a valley-dependent sign of the driving-induced mass term. A low- $k$ expansion of this expression gives the results found in $[14,17,21,22]$.

We now characterize the topology of the ac-driven lattice by explicitly calculating the Chern number from $H_{\text {eff }}(\mathbf{k})=\mathbf{h}(\mathbf{k})$. $\vec{\sigma}$, where $\vec{\sigma}$ denotes the vector of Pauli matrices. Following the elegant method of the Brouwer degree developed in Ref. [23], the Chern number (of the lower quasienergy band) reads

$$
c_{1}=\frac{1}{2} \sum_{\mathbf{D}_{i}} \operatorname{sgn}\left[\partial_{k_{x}} h_{x} \partial_{k_{y}} h_{y}-\partial_{k_{x}} h_{y} \partial_{k_{y}} h_{x}\right]_{i} \operatorname{sgn}\left[h_{z}\right]_{i} .
$$

This analytical approach requires knowing the position of the Dirac points $\mathbf{D}_{i}$ for any value of the field, which is obtained from the expression of the dressed hoppings $t_{j, 0}^{F}\left(A_{x}, A_{y}, \phi\right)$ [Eq. (4)].

We show in Fig. 1 (bottom) the values of the Chern number in the high-frequency regime as a function of the electric field amplitude $E$ and the field polarization $\phi$. To obtain the phase diagram we considered $A_{x}=A_{y}$ in the effective Hamiltonian [Eq. (5)], where the coupling between the sidebands up to first order in perturbation theory has been included. This phase diagram is the first main result of the present work. One can realize that while the Chern number remains zero for the insulating phases, it becomes \pm 1 in the perturbed SM phases. Importantly, our results also show that a change of the Chern number can originate from two different mechanisms: The first mechanism corresponds to the usual Haldane-like gap opening by TRS breaking [1] encoded into a mass term of opposite sign for the two Dirac cones. This is consistent with previous studies working within the single Dirac cone 
approximation and restricted to weak amplitudes of the driving $[12,14]$. It follows from this mechanism that a change of the chirality of the field changes the sign of the mass term $h_{z}$ in Eq. (6), thus reversing the sign of the Chern number. In contrast, the second mechanism corresponds to a change of chirality for each Dirac cone [first factor in Eq. (6)]. This happens when either the intensity or the polarization of the field is continuously varied so that a pair of Dirac cones is successively annihilated and created at two different points of the first Brillouin zone (FBZ), thus reversing their vorticity [20]. Therefore, the field polarization does not fix by itself the value of the Chern number. This leads to the counterintuitive result that a clockwise driving field can give rise to a counterclockwise edge mode, or antichiral edge state. To illustrate this mechanism, we show in Appendix $\mathrm{C}$ the quasienergy spectra for ribbons with different boundaries.

Multiphoton resonances and emergence of plateaus. We now ask whether the driving can induce gapped phases with $\left|c_{1}\right|>1$. One way to address this question is to notice that in order to obtain larger Chern numbers, it is necessary to have more than one pair of Dirac points [23]. Previous studies have shown that multiphoton resonances, occurring when the Floquet bands overlap, can induce additional pairs of Dirac points, provided that TRS holds [20,24]. Such an overlap can be achieved by decreasing the frequency, which brings the Floquet sidebands closer to each other. It is therefore natural to investigate the effect of the frequency decrease on the topology of the system for drivings that break TRS. However, a decrease in frequency increases the number of Floquet bands involved in the description of the system, and as one approaches $t \sim \omega$, the validity of $H_{\text {eff }}$ breaks down. In this regime the dynamics becomes highly nonlinear, and a numerical treatment is required.

Let us first describe how the system is affected when the sidebands overlap. In periodically driven systems, we must distinguish two inequivalent gaps: the one between the conduction and the valence bands within the same Floquet sideband $\Delta_{0}$, and the one separating two different sidebands $\Delta_{\pi}$, as depicted in Fig. 2. Note that in the high-frequency limit, the $\Delta_{\pi}$ gap was assumed to be infinite: this corresponds to the "atomic" limit between sidebands. When neighboring sidebands touch due to a frequency decrease, the gap $\Delta_{\pi}$ closes and a topological phase transition can happen.

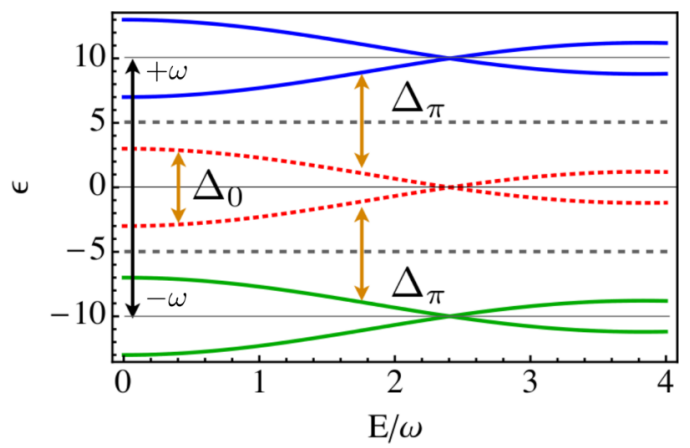

FIG. 2. (Color online) Schematic representation of three equivalent quasienergy sidebands separated by a shift $\omega$, and the two inequivalent gaps $\Delta_{0, \pi} . E$ denotes the electric field amplitude.
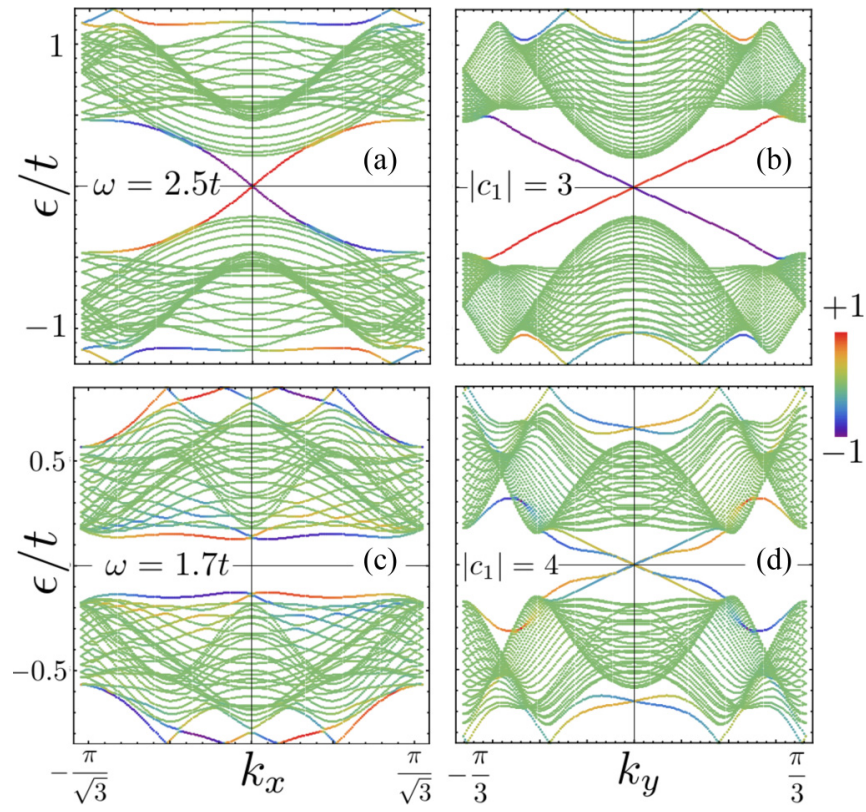

FIG. 3. (Color online) Quasienergy spectra for armchair [(a), (c)] and zigzag [(b), (d)] ribbons at $\omega=2.5 t$ (top) and $\omega=1.7 t$ (bottom), respectively. The field amplitude and polarization are $A_{x} a=A_{y} a=$ 1 and $\phi=\pi / 2$. Chiral edge states connect the valence and the conduction band. They are localized at the edges according to the time average density operator $\bar{\rho}\left(\mathbf{r}_{0}\right)-\bar{\rho}\left(\mathbf{r}_{N}\right)$ plotted in color code.

Figures 3(a) and 3(b) show the quasienergy spectra for zigzag and armchair ribbons after the gap $\Delta_{\pi}$ has closed and reopened by decreasing the frequency up to $\omega=2.5 t$. There, two chiral edge states with opposite chirality to the preexisting one in the gap $\Delta_{0}$ have emerged along the same boundary. Thus, the total chirality has changed sign whereas the field polarization has not. The system can therefore support chiral and antichiral edge states simultaneously. Importantly, the quasienergy shift of $\pm \omega / 2$ makes the states in the gap $\Delta_{\pi}$ different from the ones in the gap $\Delta_{0}$ since they contain a time-dependent correlation among the spinor components $[11,22,26]$. It is therefore important to know whether two edge states with opposite chirality lie in the same gap or not. The Chern number of this phase can be deduced from the relation $c_{1}=W_{0}-W_{\pi}=3$, where $W_{0 / \pi}$ is the total chirality of the edge states lying in the gap $\Delta_{0 / \pi}$ [8]. Figures 3(c) and $3(\mathrm{~d})$ show the previous ribbon spectra for $\omega=1.7 t$, after both $\Delta_{0, \pi}$ have closed and reopened. Note that the number of edge states crossing each gap depends on the geometry of the edge, although the total chirality in each gap $\left(W_{0, \pi}=0,4\right.$ respectively) does not. This illustrates that $W_{0, \pi}$ is another topological invariant of the system. It can actually be expressed as a $3 \mathrm{D}$ winding number in the extended $(\mathbf{k}, t)$ space [8].

Next, we use a numerical method [25] to explicitly compute $c_{1}$ for arbitrary $\omega$ and plot its (absolute) value in Fig. 4. This is the second main result of this work. It shows a plateau structure with oscillations whose period decreases with $\omega$. The oscillations are due to the coexistence of chiral and antichiral states, and reflect the dynamical topological phase transitions induced by the alternating closures and reopenings of the gaps $\Delta_{0, \pi}$ as the frequency is tuned. Importantly, for a given plateau, it is still possible to change $c_{1}$ by tuning the amplitude or the 


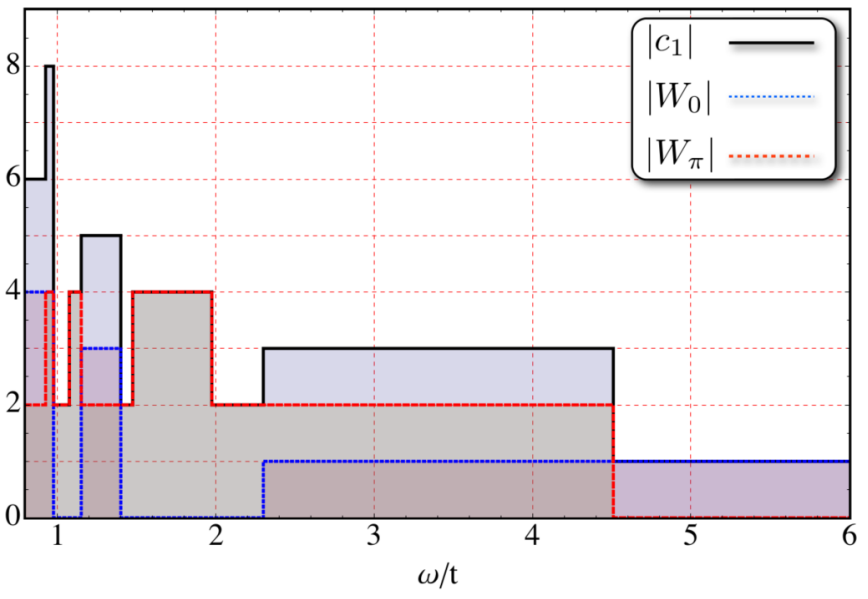

FIG. 4. (Color online) Chern number and number of chiral edge states in each gap versus the frequency $\omega$. A change in frequency creates a plateau structure in which each step is related to a closure of one of the gaps $\Delta_{0, \pi}$.

polarization of the driving, as in Fig. 1. The plateau structure of the Chern number gives information about the number of chiral edge states, but is not sufficient to fully predict the chirality at each gap. For that purpose, and for the present two-band model, one needs to know either $\left(c_{1}, W\right)$ where $W=W_{0}+W_{\pi}$, or directly $\left(W_{0}, W_{\pi}\right)$, as represented in Fig. 4.

Possible experimental realizations. Different realizations of time periodically driven honeycomb lattices can exhibit Chern number plateaus, as well as antichiral edge states. Shaken optical lattices [13] and photonic crystals with extended helical wave guides in the third spatial dimension (that simulates a circular in-plane vector potential [19]) constitute concrete physical systems with a high level of control, in which the different phases discussed here can be achieved. Microwave honeycomb crystals have also been demonstrated to be correctly described by a tight-binding model [27]. The recent realization of a Floquet microwave crystal exhibiting sidebands features [28] provides an additional promising direction for the achievement of the different regimes we describe here (the hopping parameter being of the order of a few $\mathrm{MHz}$ [27] whereas the driving frequency of the cavity can vary up to the $\mathrm{GHz}[28])$. In graphene, the possibility to induce phases with higher Chern numbers would correspond to electromagnetic frequencies $\omega / 2 \pi$ above the $\mathrm{THz}$, from the mid-infrared to visible light. The quasienergy gaps $\Delta_{0, \pi}$ and the topological edge states predicted in the present work could be probed by time-and-angle-resolved photoemission spectroscopy [29-31], as recently demonstrated on the surface of a 3D topological insulator [32]. Also, a direct connection between the edge states in the gaps $\Delta_{0, \pi}$ and the quantization of the differential conductance in a two-terminal setup has been found at high frequency in graphene [16] as well as for arbitrary frequencies in driven superconductors exhibiting Floquet Majorana particles [26,33]. It is therefore natural to expect a similar signature of the phases we find through the number of chiral and antichiral edge states in a dc transport measurement, but a rigorous treatment of this question is needed and is an important issue for future investigations.
Summary. We have proposed a general mechanism to obtain Chern phases with arbitrary values of the Chern number by periodically driving 2D Dirac semimetals. We have performed a direct calculation of the Chern number for arbitrary frequencies. Its behavior, as a function of the frequency, yields a plateau structure for the QAHE. We have also found that the Floquet Chern phases can exhibit antichiral edge modes in either of the two relevant gaps. Our model reveals as well the role of the merging and the creation of Dirac points to generate such modes. Finally we have suggested possible realizations and observations of these phases in various physical systems.

Acknowledgments: We acknowledge A. G. Grushin for useful discussions. P.D. was supported by the European Marie Curie ITN NanoCTM. Á.G.-L. acknowledges the JAE program. Á.G.-L. and G.P. acknowledge Grant No. MAT 2011-24331 for financial support.

\section{APPENDIX A: SAMBE SPACE REPRESENTATION OF THE FLOQUET OPERATOR}

For the calculation of the Floquet operator we first consider the tight-binding Hamiltonian of the undriven honeycomb lattice in reciprocal space, within the nearest-neighbor approximation, and for the basis $\left(u_{A, \mathbf{k}}, u_{B, \mathbf{k}}\right)^{T}$ :

$$
H(\mathbf{k})=t\left(\begin{array}{cc}
0 & \sum_{j=1}^{3} e^{i \mathbf{k} \cdot \mathbf{a}_{j}} \\
\sum_{j=1}^{3} e^{-i \mathbf{k} \cdot \mathbf{a}_{j}} & 0
\end{array}\right),
$$

where $\mathbf{a}_{1}=a(0,0), \mathbf{a}_{2}=a\left(\frac{3}{2}, \frac{\sqrt{3}}{2}\right), \mathbf{a}_{3}=a\left(\frac{3}{2},-\frac{\sqrt{3}}{2}\right)$. The application of the ac field is included by means of the vector potential $\mathbf{A}(\tau)$, as a time-dependent phase factor attached to the hoppings $t \rightarrow t_{j}(\tau)=t e^{i \mathbf{A}(\tau) \cdot \mathbf{d}_{j}}$, being $\mathbf{d}_{1}=a(1,0), \mathbf{d}_{2}=$ $\frac{a}{2}(-1, \sqrt{3})$, and $\mathbf{d}_{3}=\frac{a}{2}(-1,-\sqrt{3})$. Thus, the time-dependent Hamiltonian for the periodically driven honeycomb lattice is

$$
\begin{gathered}
H(\mathbf{k}, \tau)=t\left(\begin{array}{cc}
0 & \rho(\mathbf{k}, \tau) \\
\rho(\mathbf{k}, \tau)^{*} & 0
\end{array}\right), \\
\rho(\mathbf{k}, \tau) \equiv \sum_{j=1}^{3} e^{i\left[\mathbf{k} \cdot \mathbf{a}_{j}+\mathbf{A}(\tau) \cdot \mathbf{d}_{j}\right]} .
\end{gathered}
$$

The Floquet operator is given by $\mathcal{H}(\mathbf{k}, \tau)=H(\mathbf{k}, \tau)-i \partial_{\tau}$, and its representation in Sambe space leads to the matrix elements given in Eq. (1) in the main text. They are expressed in terms of the time-independent basis $\left\{\left|u_{\alpha, \mathbf{k}, p}\right\rangle\right\}$, where $p$ corresponds to the $p$ th coefficient of the Fourier expansion of $\left|u_{\alpha, \mathbf{k}}(t)\right\rangle$. Note that the importance of the Sambe space representation is that the time average included in the composed scalar product $\langle\langle\ldots\rangle\rangle=\frac{1}{T} \int_{0}^{T}\langle\ldots\rangle d t$ allows the use of the time-independent basis $\left\{\left|u_{\alpha, \mathbf{k}, p}\right\rangle\right\}$ by increasing the matrix dimension. The explicit derivation of the Floquet operator in Sambe space can be seen in Ref. [20].

\section{APPENDIX B: DERIVATION OF THE EFFECTIVE HAMILTONIAN $\boldsymbol{H}_{\text {eff }}$}

For the derivation of the effective Hamiltonian considered in Eq. (5) in the main text, we need to assume a description of the long-time dynamics $(\tau \gg 1 / \omega)$. Let us consider a stroboscopic 
evolution operator for the time-periodic Hamiltonian over a period $T$, and an approximate evolution operator described by a time-independent effective Hamiltonian $H_{\text {eff }}$ :

$$
U(T)=\mathcal{T} e^{-i \int_{0}^{T} H(\tau) d \tau} \simeq e^{-i H_{\mathrm{eff}} T} .
$$

Then, we can consider a Fourier expansion of the Hamiltonian due to its time periodicity:

$$
H(\tau)=\sum_{n=-\infty}^{\infty} H_{n} e^{i n \omega \tau} \simeq H_{0}+H_{1} e^{i \omega \tau}+H_{-1} e^{-i \omega \tau},
$$

where we have considered just the first harmonic contribution. Next, expanding the exponential in Taylor series we obtain

$$
\begin{aligned}
e^{-i \int_{0}^{T} H(\tau) d \tau} \simeq & 1-i \int_{0}^{T} H(\tau) d \tau \\
& +\frac{(-i)^{2}}{2} \int_{0}^{T} H\left(\tau_{1}\right) d \tau_{1} \int_{0}^{T} H\left(\tau_{2}\right) d \tau_{2}+\cdots .
\end{aligned}
$$

In terms of the previous expansion, the stroboscopic evolution operator is given by

$$
\begin{aligned}
U(T) \simeq \mathcal{T}\left\{1-i \int_{0}^{T} H(\tau) d \tau\right. \\
\left.+\frac{(-i)^{2}}{2} \int_{0}^{T} H\left(\tau_{1}\right) d \tau_{1} \int_{0}^{T} H\left(\tau_{2}\right) d \tau_{2}\right\} \\
=1-i \int_{0}^{T} H(\tau) d \tau-\frac{1}{2}\left[\int_{0}^{T} d \tau_{1} \int_{0}^{\tau_{1}} d \tau_{2} H\left(\tau_{1}\right) H\left(\tau_{2}\right)\right. \\
\left.+\int_{0}^{T} d \tau_{2} \int_{0}^{\tau_{2}} d \tau_{1} H\left(\tau_{2}\right) H\left(\tau_{1}\right)\right],
\end{aligned}
$$

where in the last line we have applied the time-ordering operator $\mathcal{T}$. Using Eq. (B1) we can finally perform the integrals above, and obtain the effective evolution operator:

$$
\begin{aligned}
U(T) \simeq & 1-i H_{0} T-\frac{T}{\omega}\left\{\pi H_{0}^{2}-i\left(\left[H_{0}, H_{-1}\right]\right.\right. \\
& \left.\left.-\left[H_{0}, H_{1}\right]+\left[H_{-1}, H_{1}\right]\right)\right\} \\
\simeq & 1-i H_{\mathrm{eff}} T-\frac{1}{2} H_{\mathrm{eff}}^{2} T^{2}+\cdots .
\end{aligned}
$$

By direct comparison the effective Hamiltonian reads

$$
H_{\text {eff }}=H_{0}-\frac{1}{\omega}\left(\left[H_{0}, H_{-1}\right]-\left[H_{0}, H_{1}\right]+\left[H_{-1}, H_{1}\right]\right),
$$

where we have neglected the term $H_{0}^{2}$ because it belongs to the second-order term of the Taylor expansion. It is worth mentioning that we have also derived Eq. (B2) by using the more rigorous Magnus expansion. Note that an expansion to next order yields terms which are not proportional to the Pauli matrix $\sigma_{z}$, and is therefore useless for our purposes. In addition, the gap is developed just when the $\sigma_{z}$ component of the Hamiltonian breaks TRS. This will happen when $\phi \neq 0, \pi$, since the phases attached to the hoppings in Sambe space [Eq. (2)] are nonzero. This makes the Hamiltonian to become a complex matrix, and removes the accidental degeneracies (Dirac cones) that were present in the absence of complex phases. It must also be noticed that this result is similar to the one obtained for example in [14]. Nevertheless, as we did not
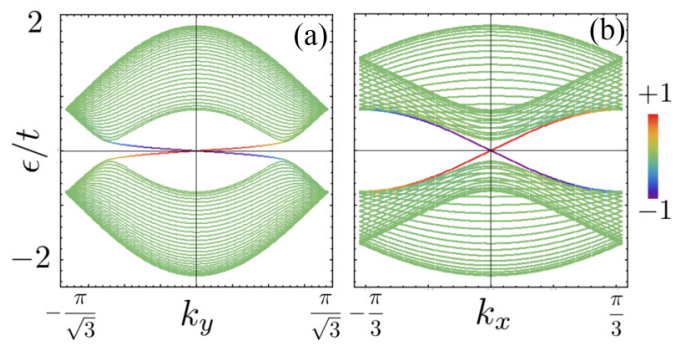

(c)

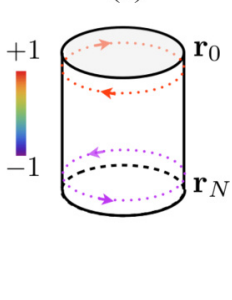

FIG. 5. (Color online) Quasienergies and edge states for the high-frequency regime $(\omega=10 t)$. (a) shows the zigzag ribbon spectrum with two chiral edge states propagating along the top/bottom (red/blue) boundary while (b) shows the armchair spectrum. In this regime $\Delta_{\pi}$ corresponds to a trivial gap, and the edge states can only cross $\Delta_{0}$. (c) shows a schematic figure of the edge states propagation. Parameters considered: 30 unit cells of length for the ribbons, $t=1$, $\phi=\pi / 2$, and $A_{x} a=A_{y} a=1$.

require a low-energy approximation to a single Dirac cone, we are not restricted to small values of the field amplitude. This feature allows us to fully address the phase diagram and the dependence of the Chern number on the external parameters.

\section{APPENDIX C: EDGE STATES}

Here we describe the properties of the different edge states appearing in our system, depending on the regime under consideration. In the high-frequency regime, the Floquet sidebands are far separated from each other; then the gap $\Delta_{\pi}$ is always trivial and does not develop topologically protected edge states (in analogy with what is usually referred as the atomic limit). Thus, all topological properties can be studied within a two-band approximation and the zero-energy modes behave equivalently to the ones of static systems. This is plotted in Fig. 5 for zigzag (a) and armchair (b) ribbons, including a color code for the time average density distribution at the edges: $\Delta \bar{\rho}=\bar{\rho}\left(\mathbf{r}_{0}\right)-\bar{\rho}\left(\mathbf{r}_{N}\right)$.

In addition, in Fig. 6 we plot the edge states chirality at each boundary for different field amplitudes and fixed phase difference $\phi=\pi / 4$. It shows how it is possible to reverse the chirality at each boundary by just tuning the amplitude of the

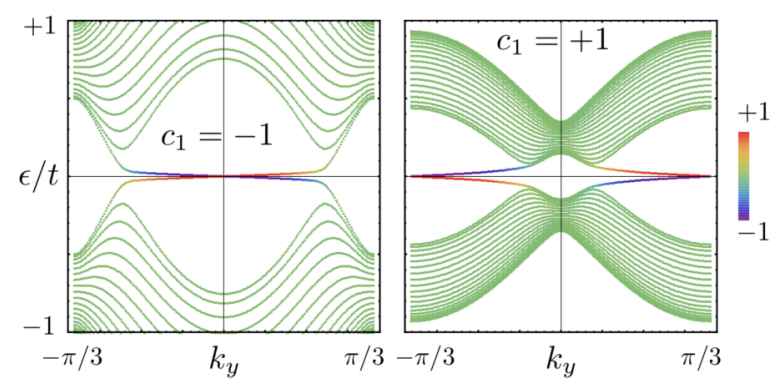

FIG. 6. (Color online) Edge states for a zigzag ribbon at different field amplitudes $A_{x} a=A_{y} a=1,3$ (left/right respectively) in the high-frequency regime $(\omega=10 t)$. The left plot shows two chiral edge states propagating along the top/bottom (red/blue) boundary, while the right plot shows the same edge states, moving in the opposite direction. Parameters considered: 20 unit cells of length for the ribbon and $\phi=\pi / 4$. 


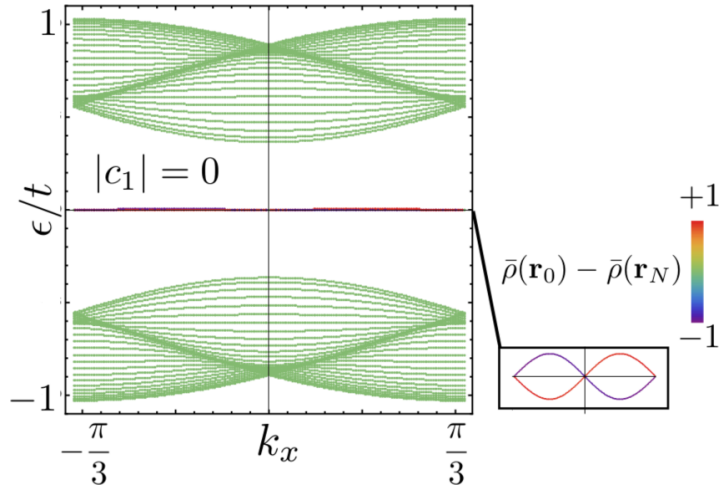

FIG. 7. (Color online) Quasienergy spectrum for graphene armchair ribbon in a topologically trivial region. The parameters are $A_{x} a=A_{y} a=2, \phi=0.6, \omega=10 t$, and 30 unit cells for the ribbon. For a zigzag ribbon nontopologically protected edge states are also found (not shown).

field, annihilating and creating a new pair of Dirac points with opposite topological charge. Since the new edge states move in opposite direction to the one dictated by the electric field polarization, we call them antichiral states.

On the other hand, when the frequency is decreased up to a certain $\omega$ value, the gap between different sidebands $\Delta_{\pi}$ collapses. This exactly happens when the frequency reaches the bandwidth value for some fixed $A_{x, y}$. When the gap $\Delta_{\pi}$ is reopened the apparition of new edge states is possible, as we show in Fig. 3 in the main text. For the case of the honeycomb lattice, the gap $\Delta_{\pi}$ develops two pairs of chiral edge states connecting different sidebands, and in consequence the Chern number changes by two units.

To conclude, when the system has a Chern number $c_{1}=$ 0 , chiral edge states do not appear (see Fig. 7). Instead, the

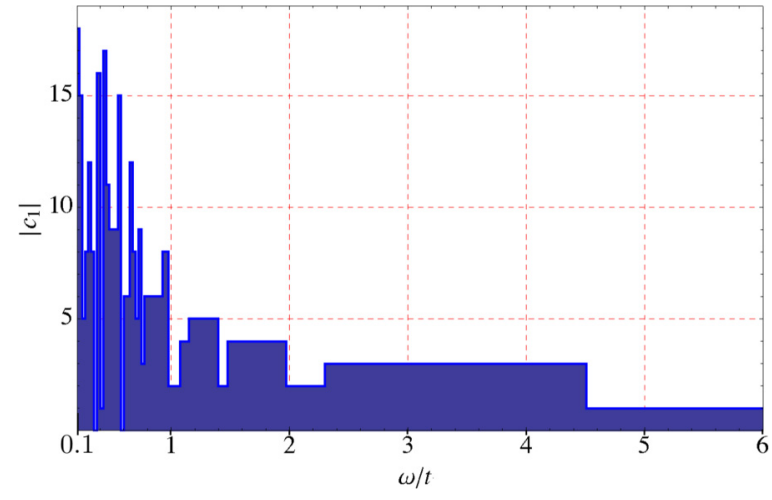

FIG. 8. (Color online) Full $\omega$ range of the numerical calculation for the Chern number (absolute value) versus the frequency in units of the hopping energy $\omega / t$. Note that the Chern number increases with the successive gap closures. The parameters considered are $A_{x} a=$ $A_{y} a=1$ and $\phi=\pi / 2$.

existence of localized end states is restricted by whether the system is in a Zak insulating phase or not. Note that these states are disconnected from the bulk and they are not dispersive. The edge states arising from a nonzero Chern number phase cross the gap, and represent a condensed matter realization of the Haldane model, where time reversal is broken for zero magnetic field.

Finally in Fig. 8 we plot the full plateau structure obtained numerically in terms of the Chern number. Note that the inequivalent bands within a single Floquet sideband in a periodically driven two-band system necessarily have opposite Chern numbers [8], so that we only need to compute the Chern number of one of them. In addition, note that the increasing number of edge states is also coincident with the one obtained in other periodically driven systems, such as in Ref. [34].
[1] F. D. M. Haldane, Phys. Rev. Lett. 61, 2015 (1988).

[2] C. L. Kane and E. J. Mele, Phys. Rev. Lett. 95, 226801 (2005).

[3] M. Konig et al., Science 318, 766 (2007).

[4] B. A. Bernevig, T. L. Hughes, and S.-C. Zhang, Science 314, 1757 (2006).

[5] C. Chang, J. Zhang, X. Feng, J. Shen, and Z. Zhang, Science 340, 167 (2013).

[6] N. Lindner, G. Refael, and V. Galitski, Nat. Phys. 7, 490 (2011).

[7] J. I. Inoue and A. Tanaka, Phys. Rev. Lett. 105, 017401 (2010).

[8] M. S. Rudner, N. H. Lindner, E. Berg, and M. Levin, Phys. Rev. X 3, 031005 (2013).

[9] A. Gómez-León and G. Platero, Phys. Rev. Lett. 110, 200403 (2013).

[10] T. Kitagawa, E. Berg, M. Rudner, and E. Demler, Phys. Rev. B 82, 235114 (2010).

[11] L. Jiang, T. Kitagawa, J. Alicea, A. R. Akhmerov, D. Pekker, G. Refael, J. I. Cirac, E. Demler, M. D. Lukin, and P. Zoller, Phys. Rev. Lett. 106, 220402 (2011).
[12] B. Dora, J. Cayssol, F. Simon, and R. Moessner, Phys. Rev. Lett. 108, 056602 (2012).

[13] P. Hauke, O. Tieleman, A. Celi, C. Ölschläger, J. Simonet, J. Struck, M. Weinberg, P. Windpassinger, K. Sengstock, M. Lewenstein, and A. Eckardt, Phys. Rev. Lett. 109, 145301 (2012).

[14] T. Kitagawa, T. Oka, A. Brataas, L. Fu, and E. Demler, Phys. Rev. B 84, 235108 (2011).

[15] A. G. Grushin, Á. Gómez-León, and T. Neupert, Phys. Rev. Lett. 112, 156801 (2014).

[16] Z. Gu, H. A. Fertig, D. P. Arovas, and A. Auerbach, Phys. Rev. Lett. 107, 216601 (2011).

[17] Y. Katan and D. Podolsky, arXiv:1309.0203.

[18] E. Suárez Morell and L. E. F. Foa Torres, Phys. Rev. B 86, 125449 (2012).

[19] M. Rechtsman et al., Nature (London) 496, 196 (2013).

[20] P. Delplace, Á. Gómez-León, and G. Platero, Phys. Rev. B 88, 245422 (2013).

[21] J. Cayssol, Phys. Status Solidi RRL 7, 101 (2013).

[22] P. M. Perez-Piskunow, G. Usaj, C. A. Balseiro, and L. E. F. Foa Torres, Phys. Rev. B 89, 121401(R) (2014). 
[23] D. Sticlet, F. Piéchon, J.-N. Fuchs, P. Kalugin, and P. Simon, Phys. Rev. B 85, 165456 (2012).

[24] B. M. Fregoso, Y. H. Wang, N. Gedik, and V. Galitski, Phys. Rev. B 88, 155129 (2013).

[25] T. Fukui, Y. Hatsugai, and H. Suzuki, J. Phys. Soc. Jpn. 74, 1674 (2005).

[26] A. Kundu, and B. Seradjeh, Phys. Rev. Lett. 111, 136402 (2013).

[27] M. Bellec, U. Kuhl, G. Montambaux, and F. Mortessagne, Phys. Rev. B 88, 115437 (2013).

[28] S. Gehler, T. Tudorovskiy, C. Schindler, U. Kuhl and H.-.J. Stockmann, New J. Phys. 15, 083030 (2013).

[29] J. A. Sobota, S. Yang, J. G. Analytis, Y. L. Chen, I. R. Fisher, P. S. Kirchmann, and Z.-X. Shen, Phys. Rev. Lett. 108, 117403 (2012).
[30] M. Hajlaoui, E. Papalazarou, J. Mauchain, G. Lantz, N. Moisan, D. Boschetto, Z. Jiang, I. Miotkowski, Y. P. Chen, A. TalebIbrahimi, L. Perfetti, and M. Marsi, Nano Lett. 12, 3532 (2012).

[31] Y. H. Wang, D. Hsieh, E. J. Sie, H. Steinberg, D. R. Gardner, Y. S. Lee, P. Jarillo-Herrero, and N. Gedik, Phys. Rev. Lett. 109, 127401 (2012).

[32] Y. H. Wang, H. Steinberg, P. Jarillo-Herrero, and N. Gedik, Science 342, 453 (2013).

[33] J. Karch, C. Drexler, P. Olbrich, M. Fehrenbacher, M. Hirmer, M. M. Glazov, S. A. Tarasenko, E. L. Ivchenko, B. Birkner, J. Eroms, D. Weiss, R. Yakimova, S. Lara-Avila, S. Kubatkin, M. Ostler, T. Seyller, and S. D. Ganichev, Phys. Rev. Lett. 107, 276601 (2011).

[34] M. Thakurathi, A. A. Patel, D. Sen, and A. Dutta, Phys. Rev. B 88, 155133 (2013). 